JOURNAL OF

FUNCTION SPACES AND APPLICATIONS

Volume 3, Number 1 (2005), 17-31
(C) 2005, Scientific Horizon http://www.jfsa.net

\title{
Equivalent norms of Herz-type Besov and Triebel-Lizorkin spaces*
}

\section{Jingshi Xu}

(Communicated by Hans Triebel)

2000 Mathematics Subject Classification. 46F05, 42B25, 42B35

Keywords and phrases. Herz space, Besov space, Triebel-Lizorkin space, Maximal function.

Abstract. In this paper the author obtains equivalent norms of Herz-type Besov and Triebel-Lizorkin spaces, which are generalizations of well-known Herztype spaces and inhomogeneous Besov and Triebel-Lizorkin spaces.

\section{Introduction}

It is well known that the theory of function spaces has played important roles in both classical analysis and modern analysis. In particular, these two scales spaces, $B_{p, q}^{s}\left(\mathbb{R}^{n}\right)$ and $F_{p, q}^{s}\left(\mathbb{R}^{n}\right)$, contain many classical spaces as special cases, for example, the Hölder spaces, the Sobolev spaces, the Besselpotential spaces, the Zygmund spaces, the local Hardy spaces and the space $\operatorname{bmo}\left(\mathbb{R}^{n}\right)$. All the above-mentioned classical spaces have been proved to be useful tools in the study of ordinary and partial differential equations. For details one can see Triebel's books [11], [12], [13] and [14].

${ }^{*}$ The project was supported by NNSF (No. 60474070) of China. 
In recent years, the Herz-type spaces have been studied by many authors. In particular, analogs of classical spaces such as Hardy spaces, Sobolev spaces and so on, have been introduced in the Herz-type setting and operators on them have been studied; see [1]-[10]. Motivated by [7], Yang and the author introduced the Herz-type Triebel-Lizorkin spaces in [15]. Some basic properties of these spaces were also given in [15], and some applications of these spaces were given in [16] and [17].

The aim of this paper is to introduce Herz-type Besov spaces. Some basic properties and applications of these spaces were already given in [18] and [19]. Before going on, we recall some notation and the definitions of Besov and Triebel-Lizorkin spaces (see, e.g., Triebel [11]).

Let $\mathbb{R}^{n}$ be the $n$-dimensional real Euclidean space and $\mathcal{S}\left(\mathbb{R}^{n}\right)$ be the Schwartz space of all complex-valued rapidly decreasing infinitely differentiable functions on $\mathbb{R}^{n}$. Let $\mathcal{S}^{\prime}\left(\mathbb{R}^{n}\right)$ be the set of all the tempered distributions on $\mathbb{R}^{n}$. If $\varphi \in \mathcal{S}\left(\mathbb{R}^{n}\right)$, then $\widehat{\varphi}$ denotes the Fourier transform of $\varphi$, and $\varphi^{\vee}$ denotes the inverse Fourier transform of $\varphi$. For $j \in \mathbb{N}$ and $x \in \mathbb{R}^{n}$ we put $\varphi_{j}(x)=2^{n j} \varphi\left(2^{j} x\right)$. Let functions $A, \theta \in \mathcal{S}\left(\mathbb{R}^{n}\right)$ satisfy the following conditions:

$$
|\widehat{A}(\xi)|>0 \text { on }\{|\xi|<2\}, \quad \text { supp } \widehat{A} \subset\{|\xi|<4\},
$$

and

$$
|\widehat{\theta}(\xi)|>0 \text { on }\{1 / 2<|\xi|<2\}, \quad \operatorname{supp} \widehat{\theta} \subset\{1 / 4<|\xi|<4\} .
$$

It is well known that Besov and Triebel-Lizorkin spaces can be defined as following.

Definition 1. (i) Let $-\infty<s<\infty, 0<q, p \leq \infty$. Then the Besov spaces $B_{p, q}^{s}\left(\mathbb{R}^{n}\right)$ are defined as all $f \in S^{\prime}\left(\mathbb{R}^{n}\right)$ such that

$$
\|f\|_{B_{p, q}^{s}}=\|A * f\|_{L_{p}}+\left\|\left\{2^{s j} \theta_{j} * f\right\}_{1}^{\infty}\right\|_{\ell_{q}\left(L_{p}\right)}<\infty .
$$

(ii) Let $-\infty<s<\infty, 0<q \leq \infty, 0<p<\infty$. Then the Triebel-Lizorkin spaces $F_{p, q}^{s}\left(\mathbb{R}^{n}\right)$ are defined as all $f \in S^{\prime}\left(\mathbb{R}^{n}\right)$ such that

$$
\|f\|_{F_{p, q}^{s}}=\|A * f\|_{L_{p}}+\left\|\left\{2^{s j} \theta_{j} * f\right\}_{1}^{\infty}\right\|_{L_{p}\left(\ell_{q}\right)}<\infty .
$$

Here $\ell_{q}\left(L_{p}\right)$ and $L_{p}\left(\ell_{q}\right)$ are the spaces of all sequences $\left\{g_{j}\right\}$ of measurable functions on $\mathbb{R}^{n}$ with finite quasi-norms

$$
\left\|\left\{g_{j}\right\}_{1}^{\infty}\right\|_{\ell_{q}\left(L_{p}\right)}=\left\|\left\{\left\|g_{j}\right\|_{L_{p}}\right\}_{1}^{\infty}\right\|_{\ell_{q}}
$$

and

$$
\left\|\left\{g_{j}\right\}_{1}^{\infty}\right\|_{L_{p}\left(\ell_{q}\right)}=\|\|\left\{g_{j}\right\}_{1}^{\infty}\left\|_{\ell_{q}}\right\|_{L_{p}} .
$$

Naturally, one can replace the Lebesgue norm by the Herz norm, then one can introduce Herz-type Besov spaces and Herz-type Triebel-Lizorkin spaces as following. 
Definition 2. (i) Let $s, \alpha \in \mathbb{R}, 0<\beta \leq \infty$, and $0<q, p \leq \infty$. Then the Herz-type Besov spaces $K_{q}^{\alpha, p} B_{\beta}^{s}\left(\mathbb{R}^{n}\right)$ and $\dot{K}_{q}^{\alpha, p} B_{\beta}^{s}\left(\mathbb{R}^{n}\right)$ are defined respectively as all $f \in S^{\prime}\left(\mathbb{R}^{n}\right)$ such that

$$
\|f\|_{K_{q}^{\alpha, p} B_{\beta}^{s}}=\|A * f\|_{K_{q}^{\alpha, p}}+\left\|\left\{2^{s j} \theta_{j} * f\right\}_{1}^{\infty}\right\|_{\ell_{\beta}\left(K_{q}^{\alpha, p}\right)}<\infty
$$

and

$$
\|f\|_{\dot{K}_{q}^{\alpha, p} B_{\beta}^{s}}=\|A * f\|_{\dot{K}_{q}^{\alpha, p}}+\left\|\left\{2^{s j} \theta_{j} * f\right\}_{1}^{\infty}\right\|_{\ell_{\beta}\left(\dot{K}_{q}^{\alpha, p}\right)}<\infty .
$$

(ii) Let $s, \alpha \in \mathbb{R}, 0<\beta \leq \infty$, and $0<q, p<\infty$. Then the Herz-type Triebel-Lizorkin spaces $K_{q}^{\alpha, p} F_{\beta}^{s}\left(\mathbb{R}^{n}\right)$ and $\dot{K}_{q}^{\alpha, p} F_{\beta}^{s}\left(\mathbb{R}^{n}\right)$ are defined respectively as all $f \in S^{\prime}\left(\mathbb{R}^{n}\right)$ such that

$$
\|f\|_{K_{q}^{\alpha, p} F_{\beta}^{s}}=\|A * f\|_{K_{q}^{\alpha, p}}+\left\|\left\{2^{s j} \theta_{j} * f\right\}_{1}^{\infty}\right\|_{K_{q}^{\alpha, p}\left(\ell_{\beta}\right)}<\infty,
$$

and

$$
\|f\|_{\dot{K}_{q}^{\alpha, p} F_{\beta}^{s}}=\|A * f\|_{\dot{K}_{q}^{\alpha, p}}+\left\|\left\{2^{s j} \theta_{j} * f\right\}_{1}^{\infty}\right\|_{\dot{K}_{q}^{\alpha, p}\left(\ell_{\beta}\right)}<\infty .
$$

Here $K_{q}^{\alpha, p}\left(\ell_{\beta}\right), \dot{K}_{q}^{\alpha, p}\left(\ell_{\beta}\right), \ell_{\beta}\left(K_{q}^{\alpha, p}\right)$ and $\ell_{\beta}\left(\dot{K}_{q}^{\alpha, p}\right)$ are defined by a way similar to those of $\ell_{q}\left(L_{p}\right)$ and $L_{p}(\ell)$ with $\|\cdot\|_{L_{p}\left(\mathbb{R}^{n}\right)}$ replaced by $\|\cdot\|_{K_{q}^{\alpha, p}}\left(\mathbb{R}^{n}\right)$ and $\|\cdot\|_{\dot{K}_{q}^{\alpha, p}}\left(\mathbb{R}^{n}\right)$; see Definition 3 for the definitions of $\|\cdot\|_{K_{q}^{\alpha, p}\left(\mathbb{R}^{n}\right)}$ and $\|\cdot\|_{\dot{K}_{q}^{\alpha, p}\left(\mathbb{R}^{n}\right)}$.

To make these space meaningful, the key point is to verify that Definition 2 is independent of the choice of functions $A$ and $\theta$. Actually, by the method of Triebel's book ([11]), we had proved that the Herz-type Triebel-Lizokin spaces is true provided $\alpha>-n / q$ in [15]. However, to prove this for the Herz-type Besov spaces, if one also follow the method in [11], one will face some difficulties. Fortunately, recently in [8], Rychkov obtained a stronger result than that in [11]. In Section 3 we will prove that Definition 2 is independent of the choice of functions $A, \theta$. In fact, we have the following theorem, which is stronger than what we need and gives a unified approach for Herz-type Besov spaces and Herz-type Triebel-Lizorkin spaces.

Let $\Psi, \psi \in \mathcal{S}\left(\mathbb{R}^{n}\right), \epsilon>0$ and integer $S \geq-1$ be such that

$$
\begin{gathered}
|\widehat{\Psi}(\xi)|>0 \text { on }\{|\xi|<2 \epsilon\}, \\
|\widehat{\psi}(\xi)|>0 \text { on }\{\epsilon / 2<|\xi|<2 \epsilon\},
\end{gathered}
$$

and

$$
D^{\tau} \widehat{\psi}(0)=0 \text { for all }|\tau| \leq S .
$$

Here (1) are Tauberian conditions, while (2) expresses moment conditions on $\psi$. 
20 Equivalent norms of Herz-type Besov and Triebel-Lizorkin spaces

For any $a>0, f \in \mathcal{S}^{\prime}\left(\mathbb{R}^{n}\right)$, and $x \in \mathbb{R}^{n}$, we define the maximal functions,

$$
\Psi_{a}^{*} f(x)=\sup _{y \in \mathbb{R}^{n}} \frac{|\Psi * f(y)|}{(1+|x-y|)^{a}},
$$

and

$$
\psi_{j, a}^{*} f(x)=\sup _{y \in \mathbb{R}^{n}} \frac{\left|\psi_{j} * f(y)\right|}{\left(1+2^{j}|x-y|\right)^{a}} .
$$

In what follows, the symbol $\lesssim$ means an estimate with a positive constant independent of $f \in \mathcal{S}^{\prime}\left(\mathbb{R}^{n}\right)$.

Theorem 1. (i) Let $s<S+1,0<\beta \leq \infty, 0<q, p \leq \infty, \alpha>-n / q$, and $a>n / q$. Then for all $f \in \mathcal{S}^{\prime}\left(\mathbb{R}^{n}\right)$

$$
\begin{aligned}
\left\|\Psi_{a}^{*} f\right\|_{K_{q}^{\alpha, p}}+ & \left\|\left\{2^{s j} \psi_{j, a}^{*} f\right\}_{1}^{\infty}\right\|_{\ell_{\beta}\left(K_{q}^{\alpha, p}\right)} \\
& \lesssim\|f\|_{K_{q}^{\alpha, p} B_{\beta}^{s}} \\
& \lesssim\|\Psi * f\|_{K_{q}^{\alpha, p}}+\left\|\left\{2^{j s} \psi_{j} * f\right\}_{1}^{\infty}\right\|_{\ell_{\beta}\left(K_{q}^{\alpha, p}\right)},
\end{aligned}
$$

and

$$
\begin{aligned}
\left\|\Psi_{a}^{*} f\right\|_{\dot{K}_{q}^{\alpha, p}}+ & \left\|\left\{2^{s j} \psi_{j, a}^{*} f\right\}_{1}^{\infty}\right\|_{\ell_{\beta}\left(\dot{K}_{q}^{\alpha, p}\right)} \\
& \lesssim\|f\|_{\dot{K}_{q}^{\alpha, p} B_{\beta}^{s}} \\
& \lesssim\|\Psi * f\|_{\dot{K}_{q}^{\alpha, p}}+\left\|\left\{2^{j s} \psi_{j} * f\right\}_{1}^{\infty}\right\|_{\ell_{\beta}\left(\dot{K}_{q}^{\alpha, p}\right)} .
\end{aligned}
$$

(ii) Let $s<S+1,0<\beta \leq \infty, 0<q$, $p<\infty, \alpha>-n / q$, and $a>n / \min (q, \beta)$. Then for all $f \in \mathcal{S}^{\prime}\left(\mathbb{R}^{n}\right)$

$$
\begin{aligned}
\left\|\Psi_{a}^{*} f\right\|_{K_{q}^{\alpha, p}}+ & \left\|\left\{2^{s j} \psi_{j, a}^{*} f\right\}_{1}^{\infty}\right\|_{K_{q}^{\alpha, p}\left(\ell_{\beta}\right)} \\
& \lesssim\|f\|_{K_{q}^{\alpha, p} F_{\beta}^{s}} \\
& \lesssim\|\Psi * f\|_{K_{q}^{\alpha, p}}+\left\|\left\{2^{j s} \psi_{j} * f\right\}_{1}^{\infty}\right\|_{K_{q}^{\alpha, p}\left(\ell_{\beta}\right)},
\end{aligned}
$$

and

$$
\begin{aligned}
\left\|\Psi_{a}^{*} f\right\|_{\dot{K}_{q}^{\alpha, p}}+ & \left\|\left\{2^{s j} \psi_{j, a}^{*} f\right\}_{1}^{\infty}\right\|_{\dot{K}_{q}^{\alpha, p}\left(\ell_{\beta}\right)} \\
& \lesssim\|f\|_{\dot{K}_{q}^{\alpha, p} F_{\beta}^{s}} \\
& \lesssim\|\Psi * f\|_{\dot{K}_{q}^{\alpha, p}}+\left\|\left\{2^{j s} \psi_{j} * f\right\}_{1}^{\infty}\right\|_{\dot{K}_{q}^{\alpha, p}\left(\ell_{\beta}\right)} .
\end{aligned}
$$

Remark. In what follows, $C$ denotes a positive constant, which is independent of the main parameters, but may vary from line to line. A constant with subscripts means that it depends the parameters indicated by the subscripts. By writing $A_{1} \lesssim A_{2}$ we mean that there is a constant $C>0$ such that $A_{1} \leq C A_{2}$. In the sequel, for convenience, we also omit the range of integration when it is $\mathbb{R}^{n}$. 


\section{Some lemmas}

Let $Q_{k}=\left\{x \in \mathbb{R}^{n}:|x| \leq 2^{k}\right\}$ and $D_{k}=Q_{k} \backslash Q_{k-1}$ for $k \in \mathbb{Z}$. If $E$ is a subset of $\mathbb{R}^{n}$, we let $\chi_{E}$ denote the characteristic function of the set $E$. For convenience, we denote $\chi_{D_{k}}$ simply by $\chi_{k}$.

Definition 3 (see [2]). Let $\alpha \in \mathbb{R}$ and $0<p, q \leq \infty$.

(i) The homogeneous Herz space $\dot{K}_{q}^{\alpha, p}\left(\mathbb{R}^{n}\right)$ is defined in terms of

$$
\|f\|_{\dot{K}_{q}^{\alpha, p}}=\left\{\sum_{k=-\infty}^{\infty} 2^{k \alpha p}\left\|f \chi_{k}\right\|_{L^{q}}^{p}\right\}^{1 / p}
$$

by letting

$$
\dot{K}_{q}^{\alpha, p}\left(\mathbb{R}^{n}\right)=\left\{f \in L_{l o c}^{q}\left(\mathbb{R}^{n} \backslash\{0\}\right):\|f\|_{\dot{K}_{q}^{\alpha, p}}<\infty\right\} .
$$

(ii) The non-homogeneous Herz space $K_{q}^{\alpha, p}\left(\mathbb{R}^{n}\right)$ is defined in terms of

$$
\|f\|_{K_{q}^{\alpha, p}}=\left\{\left\|f \chi_{Q_{0}}\right\|_{L^{q}}^{p}+\sum_{k=1}^{\infty} 2^{k \alpha p}\left\|f \chi_{k}\right\|_{L^{q}}^{p}\right\}^{1 / p}
$$

by letting

$$
K_{q}^{\alpha, p}\left(\mathbb{R}^{n}\right)=\left\{f \in L_{l o c}^{q}\left(\mathbb{R}^{n}\right):\|f\|_{K_{q}^{\alpha, p}}<\infty\right\} .
$$

Here the usual modifications are made when $p=\infty$ or $q=\infty$.

Lemma 1 (see [8]). Let $\mu, \nu \in \mathcal{S}\left(\mathbb{R}^{n}\right)$, integer $M \geq-1$ and

$$
D^{\tau} \widehat{\mu}(0)=0 \text { for all }|\tau| \leq M .
$$

Then for any $N>0$ there is a constant $C_{N}>0$ such that

$$
\sup _{x \in \mathbb{R}^{n}}\left|\mu_{t} * \nu(z)\right|(1+|z|)^{N} \leq C_{N} t^{M+1} .
$$

The following Lemma 2 is easy to obtain. For its proof one can also see [8].

Lemma 2. Let $0<\beta \leq \infty$, and $\delta>0$. For any sequence $\left\{g_{j}\right\}_{0}^{\infty}$ of nonnegative measurable functions on $\mathbb{R}^{n}$, set

$$
G_{j}(x)=\sum_{k=0}^{\infty} 2^{-|k-j|^{\delta}} g_{k}(x), \quad x \in \mathbb{R}^{n} .
$$

Then

$$
\left\|\left\{G_{j}(x)\right\}_{0}^{\infty}\right\|_{\ell_{\beta}} \leq C\left\|\left\{g_{j}(x)\right\}_{0}^{\infty}\right\|_{\ell_{\beta}}
$$

holds, where $C>0$ is a constant only dependent on $\beta$ and $\delta$. 
22 Equivalent norms of Herz-type Besov and Triebel-Lizorkin spaces

Lemma 3. Let $0<p, q, \beta \leq \infty$, and $\delta>0$. For any sequence $\left\{g_{j}\right\}_{0}^{\infty}$ of nonnegative measurable functions on $\mathbb{R}^{n}$, set

$$
G_{j}(x)=\sum_{k=0}^{\infty} 2^{-|k-j|^{\delta}} g_{k}(x), \quad x \in \mathbb{R}^{n} .
$$

Then

$$
\left\|\left\{G_{j}\right\}_{0}^{\infty}\right\|_{K_{q}^{\alpha, p}\left(\ell_{\beta}\right)} \leq C_{1}\left\|\left\{g_{j}\right\}_{0}^{\infty}\right\|_{K_{q}^{\alpha, p}\left(\ell_{\beta}\right)}
$$

and

$$
\left\|\left\{G_{j}\right\}_{0}^{\infty}\right\|_{\ell_{\beta}\left(K_{q}^{\alpha, p}\right)} \leq C_{2}\left\|\left\{g_{j}\right\}_{0}^{\infty}\right\|_{\ell_{\beta}\left(K_{q}^{\alpha, p}\right)}
$$

hold with some positive constants $C_{1}=C_{1}(\beta, \delta)$ and $C_{2}=C_{2}(p, q, \beta, \delta)$. There is a similar result if one replace $K_{q}^{\alpha, p}$ by $\dot{K}_{q}^{\alpha, p}$.

Proof. By Lemma 2, we can deduce (7) follows from (6). Now we prove (8) by considering four cases.

Case 1. $p, q \geq 1$. Since $\|\cdot\|_{K_{q}^{\alpha, p}}$ is a norm, by Minkowski's inequality, we have

$$
\left\|G_{j}\right\|_{K_{q}^{\alpha, p}} \leq \sum_{k=0}^{\infty} 2^{-|k-j| \delta}\left\|g_{k}\right\|_{K_{q}^{\alpha, p}} .
$$

Hence (8) follows from Lemma 2.

Case 2. $q \leq 1, p<1$. In this case, we have

$$
\begin{aligned}
\left\|G_{j}\right\|_{K_{q}^{\alpha, p}}^{p} & =\sum_{l=0}^{\infty} 2^{l \alpha p}\left\|G_{j} \chi_{l}\right\|_{L^{q}}^{p} \\
& \leq \sum_{l=0}^{\infty} 2^{l \alpha p}\left(\sum_{k=0}^{\infty} 2^{-|k-j| \delta}\left\|g_{k} \chi_{l}\right\|_{L^{q}}\right)^{p} \\
& \leq \sum_{l=0}^{\infty} 2^{l \alpha p} \sum_{k=0}^{\infty} 2^{-p|k-j| \delta}\left\|g_{k} \chi_{l}\right\|_{L^{q}}^{p} \\
& =\sum_{k=0}^{\infty} 2^{-|k-j| p \delta} \sum_{l=0}^{\infty} 2^{l \alpha p}\left\|g_{k} \chi_{l}\right\|_{L^{q}}^{p} \\
& =\sum_{k=0}^{\infty} 2^{-|k-j| p \delta}\left\|g_{k}\right\|_{K_{q}^{\alpha, p}}^{p} .
\end{aligned}
$$

By Lemma 2 with $\beta$ and $\delta$ replaced by $\beta / p$ and $p \delta$ respectively, we have

$$
\left\|\left\{\left\|G_{j}\right\|_{K_{q}^{\alpha, p}}^{p}\right\}\right\|_{\ell_{\beta / p}} \leq C\left\|\left\{\left\|g_{j}\right\|_{K_{q}^{\alpha, p}}^{p}\right\}\right\|_{\ell_{\beta / p}} .
$$

Raising the above inequality to power $1 / p$, we obtain (8). 
Case 3. $q, p<1$, and $p / q \leq 1$. First, by Minkowski's inequality, we have

$$
\left\|G_{j}\right\|_{K_{q}^{\alpha, p}}^{p}=\sum_{l=0}^{\infty} 2^{l \alpha p}\left\|G_{j} \chi_{l}\right\|_{L^{q}}^{p} \leq \sum_{l=0}^{\infty} 2^{l \alpha p}\left(\sum_{k=0}^{\infty} 2^{-|k-j| q \delta} \int g_{k}^{q} \chi_{l} d x\right)^{p / q} .
$$

Then,

$$
\begin{aligned}
\left\|\left\{\left\|G_{j}\right\|_{K_{q}^{\alpha, p}}^{p}\right\}\right\|_{\ell_{\beta} / p}^{\beta / p} & \leq \sum_{j=0}^{\infty}\left[\sum_{l=0}^{\infty} 2^{l \alpha p}\left(\sum_{k=0}^{\infty} 2^{-|k-j| q \delta} \int g_{k}^{q} \chi_{l} d x\right)^{p / q}\right]^{\beta / p} \\
& \leq \sum_{j=0}^{\infty}\left[\sum_{l=0}^{\infty} 2^{l \alpha p} \sum_{k=0}^{\infty} 2^{-|k-j| p \delta}\left(\int g_{k}^{q} \chi_{l} d x\right)^{p / q}\right]^{\beta / p} \\
& \leq \sum_{j=0}^{\infty}\left[\sum_{k=0}^{\infty} 2^{-|k-j| p \delta} \sum_{l=0}^{\infty} 2^{l \alpha p}\left(\int g_{k}^{q} \chi_{l} d x\right)^{p / q}\right]^{\beta / p} \\
& \leq \sum_{j=0}^{\infty}\left[\sum_{k=0}^{\infty} 2^{-|k-j| p \delta}\left\|g_{k}\right\|_{K_{q}^{\alpha, p}}^{p}\right]^{\beta / p} \\
& \leq C\left[\sum_{k=0}^{\infty}\left\|g_{k}\right\|_{K_{q}^{\alpha, p}}^{\beta}\right]^{p / \beta},
\end{aligned}
$$

where the last inequality used Lemma 2 with $\beta$ and $\delta$ replaced by $\beta / p$ and $\delta p$ respectively. Raising to the power $1 / p$, we obtain (8).

Case 4. $q, p \leq 1$, and $p / q>1$. In this case

$$
\begin{aligned}
\left\|\left\{\left\|G_{j}\right\|_{K_{q}^{\alpha, p}}^{p}\right\}\right\|_{\ell_{\beta} / p}^{\beta / p} & \leq \sum_{j=0}^{\infty}\left[\sum_{l=0}^{\infty} 2^{l \alpha p}\left(\sum_{k=0}^{\infty} 2^{-|k-j| q \delta} \int g_{k}^{q} \chi_{l} d x\right)^{p / q}\right]^{\beta / p} \\
\leq & \sum_{j=0}^{\infty}\left[\sum_{l=0}^{\infty} 2^{l \alpha p}\left(\sum_{k=0}^{\infty} 2^{-|k-j| q \delta_{1} u}\right)^{p / u q}\right. \\
& \left.\times\left(\sum_{k=0}^{\infty} 2^{-|k-j| p \delta_{2}}\left(\int g_{k}^{q} \chi_{l} d x\right)^{p / q}\right)\right]^{\beta / p} \\
\leq & C \sum_{j=0}^{\infty}\left[\sum_{k=0}^{\infty} 2^{-|k-j| p \delta_{2}} \sum_{l=0}^{\infty} 2^{l \alpha p}\left(\int g_{k}^{q} \chi_{l} d x\right)^{p / q}\right]^{\beta / p} \\
\leq & C \sum_{j=0}^{\infty}\left[\sum_{k=0}^{\infty} 2^{-|k-j| p \delta_{2}}\left\|g_{k}\right\|_{K_{q}^{\alpha, p}}^{p}\right]^{\beta / p} \\
\leq & C\left[\sum_{k=0}^{\infty}\left\|g_{k}\right\|_{K_{q}^{\alpha, p}}^{\beta}\right]^{p / \beta}
\end{aligned}
$$


24 Equivalent norms of Herz-type Besov and Triebel-Lizorkin spaces

where $\delta_{1}+\delta_{2}=\delta, \delta_{1}, \delta_{2}>0,1 / u+q / p=1$; in the second inequality we used the Hölder inequality and in the last inequality we used Lemma 2 with $\beta$ and $\delta$ replaced by $\beta / p$ and $p \delta_{2}$ respectively. Raising to the power $1 / p$, we obtain (8) again.

This completes the proof of (8) for the spaces $K_{q}^{\alpha, p}\left(\mathbb{R}^{n}\right)$. The proof of (8) for the spaces $\dot{K}_{q}^{\alpha, p}\left(\mathbb{R}^{n}\right)$ is similar, and we omit the detail.

Lemma 4 (see [10]). Let $1<\beta \leq \infty, 1<q<\infty$ and $0<p<\infty$. If $\left\{f_{j}\right\}_{j=0}^{\infty}$ is a sequence of locally integrable functions on $\mathbb{R}^{n}$ and $-n / q<\alpha<$ $n(1-1 / q)$, then

$$
\left\|\left\{\mathcal{M}\left(f_{j}\right)\right\}\right\|_{K_{q}^{\alpha, p}\left(\ell_{\beta}\right)} \leq C\left\|\left\{f_{j}\right\}\right\|_{K_{q}^{\alpha, p}\left(\ell_{\beta}\right)}
$$

and

$$
\left\|\left\{\mathcal{M}\left(f_{j}\right)\right\}\right\|_{\dot{K}_{q}^{\alpha, p}\left(\ell_{\beta}\right)} \leq C\left\|\left\{f_{j}\right\}\right\|_{\dot{K}_{q}^{\alpha, p}\left(\ell_{\beta}\right)},
$$

where $C>0$ is a constant independent of $\left\{f_{j}\right\}_{j=0}^{\infty}$ and $\mathcal{M}$ denotes the Hardy-Littlewood maximal operator.

Lemma 5 (see [8]). Let $0<r \leq 1$, and $\left\{b_{j}\right\}_{0}^{\infty}$ and $\left\{d_{j}\right\}_{0}^{\infty}$ be two sequences taking values in $(0,+\infty]$ and $(0,+\infty)$ respectively. Assume that for some $N_{0}>0$,

$$
d_{j}=O\left(2^{j N_{0}}\right), \quad j \rightarrow \infty
$$

and that for any $N>0$, and $j \in \mathbb{N}_{0}=\mathbb{N} \cup\{0\}$, there exists a positive constant $C_{N}$ independent of $j$ such that

$$
d_{j} \leq C_{N} \sum_{k=j}^{\infty} 2^{(j-k) N} b_{k} d_{k}^{1-r} .
$$

Then for any $N>0$ and $j \in \mathbb{N}_{0}$,

$$
d_{j}^{r} \leq C_{N} \sum_{k=j}^{\infty} 2^{(j-k) N r} b_{k}
$$

holds with the same constant $C_{N}$ as above.

\section{Proof of Theorem 1}

The main idea of the proof comes from Rychkov [8]. In fact we will prove Theorem 1 by a procedure similar to that of Theorem 1 in [8] with 3 steps. Step 1. Take any pair of functions $\Phi, \varphi \in \mathcal{S}\left(\mathbb{R}^{n}\right)$ so that for an $\varepsilon^{\prime}>0$

$$
\begin{array}{ll}
|\widehat{\Phi}(\xi)|>0 & \text { on }\left\{|\xi|<2 \varepsilon^{\prime}\right\}, \\
|\widehat{\varphi}(\xi)|>0 & \text { on }\left\{\varepsilon^{\prime} / 2<|\xi|<2 \varepsilon^{\prime}\right\},
\end{array}
$$

and define $\Phi_{a}^{*} f, \varphi_{j, a}^{*} f$ as $(3)$ and $\left(3^{\prime}\right)$. 
For any $a>0, s<S+1$, and $0<p, q, \beta \leq \infty$, we will prove that for all $f \in \mathcal{S}^{\prime}\left(\mathbb{R}^{n}\right)$ the following estimates hold.

$$
\begin{gathered}
\left\|\Psi_{a}^{*} f\right\|_{K_{q}^{\alpha, p}}+\left\|\left\{2^{s j} \psi_{j, a}^{*} f\right\}_{1}^{\infty}\right\|_{\ell_{\beta}\left(K_{q}^{\alpha, p}\right)} \\
\quad\|\| \Phi_{a}^{*} f\left\|_{K_{q}^{\alpha, p}}+\right\|\left\{2^{j s} \varphi_{j, a}^{*} f\right\}_{1}^{\infty} \|_{\ell_{\beta}\left(K_{q}^{\alpha, p}\right)}, \\
\left\|\Psi_{a}^{*} f\right\|_{\dot{K}_{q}^{\alpha, p}}+\left\|\left\{2^{s j} \psi_{j, a}^{*} f\right\}_{1}^{\infty}\right\|_{\ell_{\beta}\left(\dot{K}_{q}^{\alpha, p}\right)} \\
\quad \lesssim\left\|\Phi_{a}^{*} f\right\|_{\dot{K}_{q}^{\alpha, p}}+\left\|\left\{2^{j s} \varphi_{j, a}^{*} f\right\}_{1}^{\infty}\right\|_{\ell_{\beta}\left(\dot{K}_{q}^{\alpha, p}\right)}, \\
\left\|\Psi_{a}^{*} f\right\|_{K_{q}^{\alpha, p}}+\left\|\left\{2^{s j} \psi_{j, a}^{*} f\right\}_{1}^{\infty}\right\|_{K_{q}^{\alpha, p}\left(\ell_{\beta}\right)} \\
\lesssim\left\|\Phi_{a}^{*} f\right\|_{K_{q}^{\alpha, p}}+\left\|\left\{2^{j s} \varphi_{j, a}^{*} f\right\}_{1}^{\infty}\right\|_{K_{q}^{\alpha, p}\left(\ell_{\beta}\right)},
\end{gathered}
$$

and

$$
\begin{aligned}
\left\|\Psi_{a}^{*} f\right\|_{\dot{K}_{q}^{\alpha, p}}+\| & \left\{2^{s j} \psi_{j, a}^{*} f\right\}_{1}^{\infty} \|_{\dot{K}_{q}^{\alpha, p}\left(\ell_{\beta}\right)} \\
& \lesssim\left\|\Phi_{a}^{*} f\right\|_{\dot{K}_{q}^{\alpha, p}}^{\alpha}+\left\|\left\{2^{j s} \varphi_{j, a}^{*} f\right\}_{1}^{\infty}\right\|_{\dot{K}_{q}^{\alpha, p}\left(\ell_{\beta}\right)}
\end{aligned}
$$

In fact, it follows from (9) that there exist two functions $\Lambda, \lambda \in \mathcal{S}\left(\mathbb{R}^{n}\right)$ such that

$$
\begin{gathered}
\operatorname{supp} \widehat{\Lambda} \subset\left\{|\xi|<2 \varepsilon^{\prime}\right\}, \\
\operatorname{supp} \hat{\lambda} \subset\left\{\varepsilon^{\prime} / 2<|\xi|<2 \varepsilon^{\prime}\right\},
\end{gathered}
$$

and

$$
\widehat{\Lambda}(\xi) \widehat{\Phi}(\xi)+\sum_{j=1}^{\infty} \widehat{\lambda}\left(2^{-j} \xi\right) \widehat{\varphi}\left(2^{-j} \xi\right) \equiv 1 \quad \text { for all } \xi \in \mathbb{R}^{n} .
$$

Then, for all $f \in \mathcal{S}^{\prime}\left(\mathbb{R}^{n}\right)$, we have the identity,

$$
f=\Lambda * \Phi * f+\sum_{k=1}^{\infty} \lambda_{k} * \psi_{k} * f .
$$

Thus we can write

$$
\psi_{j} * f=\psi_{j} * \Lambda * \Phi * f+\sum_{k=1}^{\infty} \psi_{j} * \lambda_{k} * \psi_{k} * f .
$$

Therefore, by Lemma 1,

$$
\begin{aligned}
\left|\psi_{j} * \lambda_{k} * \varphi_{k} * f(y)\right| & \leq \int_{\mathbb{R}^{n}}\left|\psi_{j} * \lambda_{k}(z) \| \varphi_{k} * f(y-z)\right| d z \\
& \leq \varphi_{k, a}^{*} f(y) \int_{\mathbb{R}^{n}}\left|\psi_{j} * \lambda_{k}(z)\right| \mid\left(1+2^{k}|z|\right)^{a} d z \\
& \equiv \varphi_{k, a}^{*} f(y) I_{j, k}
\end{aligned}
$$

where

$$
I_{j, k} \leq C(\lambda, \psi) \begin{cases}2^{(k-j)(S+1)}, & \text { if } \quad k \leq j \\ 2^{(j-k)(S+1)}, & \text { if } \quad k \geq j\end{cases}
$$


26 Equivalent norms of Herz-type Besov and Triebel-Lizorkin spaces

see [8]. Noting that for all $x, y \in \mathbb{R}^{n}$,

$\varphi_{k, a}^{*} f(y) \leq \varphi_{k, a}^{*} f(x)\left(1+2^{k}|x-y|\right)^{a} \leq \varphi_{k, a}^{*} f(x) \max \left(1,2^{(k-j) a}\right)\left(1+2^{j}|x-y|\right)^{a}$.

So we have

$$
\sup _{y \in \mathbb{R}^{n}} \frac{\left|\psi_{j} * \lambda_{k} * \varphi_{k} * f(y)\right|}{\left(1+2^{j}|x-y|\right)^{a}} \lesssim \varphi_{k, a}^{*} f(x) \times \begin{cases}2^{(k-j)(S+1)}, & \text { if } \quad k \leq j \\ 2^{(j-k)(S+1)}, & \text { if } \quad k \geq j\end{cases}
$$

Note that for $k=1$, we do not use the condition $D^{\tau} \hat{\lambda}(0)=0$ in the above proof of the last estimate, so by replacing respectively $\lambda_{1}$ and $\varphi_{1}$ with $\Lambda$ and $\Phi$, we have a similar estimate

$$
\sup _{y \in \mathbb{R}^{n}} \frac{\left|\psi_{j} * \Lambda * \varphi_{k} * f(y)\right|}{\left(1+2^{j}|x-y|\right)^{a}} \lesssim \Phi_{a}^{*} f(x) 2^{-j(S+1)} .
$$

So we obtain

$$
\psi_{j, a}^{*} f(x) \lesssim \Phi_{a}^{*} f(x) 2^{-j(S+1)}+\sum_{k=1}^{\infty} \varphi_{k, a}^{*} f(x) \times \begin{cases}2^{(k-j)(S+1)}, & \text { if } \quad k \leq j \\ 2^{(j-k)(S+1)}, & \text { if } \quad k \geq j\end{cases}
$$

Hence with $\delta=\min (1, S+1-s)>0$ for all $f \in \mathcal{S}^{\prime}, x \in \mathbb{R}^{n}, j \in \mathbb{N}$,

$$
2^{j s} \psi_{j, a}^{*} f(x) \lesssim \Phi_{a}^{*} f(x) 2^{-j \delta}+\sum_{k=1}^{\infty} 2^{k s} \varphi_{k, a}^{*} f(x) 2^{-|k-j| \delta} .
$$

Again, for $j=1$ we did not use (2) to obtain this estimate, so we can replace $\psi_{1}$ with $\Psi$ to obtain

$$
2^{j s} \Psi_{a}^{*} f(x) \lesssim \Phi_{a}^{*} f(x) 2^{-j \delta}+\sum_{k=1}^{\infty} 2^{k s} \varphi_{k, a}^{*} f(x) 2^{-j \delta} .
$$

The desired estimates (10), (10'), (11), (11') follow from (12), (13) and Lemma 3.

Step 2. In this step we will show the following estimates.

In the conditions of (4), for all $f \in \mathcal{S}^{\prime}(\mathbb{R})$

$$
\begin{aligned}
\left\|\Psi_{a}^{*} f\right\|_{K_{q}^{\alpha, p}}+\left\|\left\{2^{s j} \psi_{j, a}^{*} f\right\}_{1}^{\infty}\right\|_{\ell_{\beta}\left(K_{q}^{\alpha, p}\right)} & \\
& \lesssim\|\Psi * f\|_{K_{q}^{\alpha, p}}+\left\|\left\{2^{j s} \psi_{j} * f\right\}_{1}^{\infty}\right\|_{\ell_{\beta}\left(K_{q}^{\alpha, p}\right)}
\end{aligned}
$$

and

$$
\begin{aligned}
\left\|\Psi_{a}^{*} f\right\|_{\dot{K}_{q}^{\alpha, p}}+\left\|\left\{2^{s j} \psi_{j, a}^{*} f\right\}_{1}^{\infty}\right\|_{\ell_{\beta}\left(\dot{K}_{q}^{\alpha, p}\right)} & \\
& \lesssim\|\Psi * f\|_{\dot{K}_{q}^{\alpha, p}}+\left\|\left\{2^{j s} \psi_{j} * f\right\}_{1}^{\infty}\right\|_{\ell_{\beta}\left(\dot{K}_{q}^{\alpha, p}\right)}
\end{aligned}
$$

In the conditions of $(5)$, for all $f \in \mathcal{S}^{\prime}\left(\mathbb{R}^{n}\right)$

$$
\begin{aligned}
\left\|\Psi_{a}^{*} f\right\|_{K_{q}^{\alpha, p}}+\| & \left\{2^{s j} \psi_{j, a}^{*} f\right\}_{1}^{\infty} \|_{K_{q}^{\alpha, p}\left(\ell_{\beta}\right)} \\
& \lesssim\|\Psi * f\|_{K_{q}^{\alpha, p}}+\left\|\left\{2^{j s} \psi_{j} * f\right\}_{1}^{\infty}\right\|_{K_{q}^{\alpha, p}\left(\ell_{\beta}\right)}
\end{aligned}
$$


and

$$
\begin{aligned}
\left\|\Psi_{a}^{*} f\right\|_{\dot{K}_{q}^{\alpha, p}}+\left\|\left\{2^{s j} \psi_{j, a}^{*} f\right\}_{1}^{\infty}\right\|_{\dot{K}_{q}^{\alpha, p}\left(\ell_{\beta}\right)} & \\
& \lesssim\|\Psi * f\|_{\dot{K}_{q}^{\alpha, p}}+\left\|\left\{2^{j s} \psi_{j} * f\right\}_{1}^{\infty}\right\|_{\dot{K}_{q}^{\alpha, p}\left(\ell_{\beta}\right)} .
\end{aligned}
$$

Similar to (9), choose two functions $\Lambda, \lambda \in \mathcal{S}\left(\mathbb{R}^{n}\right)$ such that

$$
\begin{gathered}
\operatorname{supp} \hat{\Lambda} \subset\left\{|\xi|<2 \varepsilon^{\prime}\right\}, \\
\operatorname{supp} \hat{\lambda} \subset\left\{\varepsilon^{\prime} / 2<|\xi|<2 \varepsilon^{\prime}\right\},
\end{gathered}
$$

and

$$
\widehat{\Lambda}(\xi) \widehat{\Phi}(\xi)+\sum_{j=1}^{\infty} \widehat{\lambda}\left(2^{-j} \xi\right) \widehat{\varphi}\left(2^{-j} \xi\right) \equiv 1
$$

for all $\xi \in \mathbb{R}^{n}$. Then, for all $f \in \mathcal{S}^{\prime}\left(\mathbb{R}^{n}\right)$, we have the identity,

$$
f=\Lambda * \Phi * f+\sum_{k=1}^{\infty} \lambda_{k} * \psi_{k} * f .
$$

Thus we can write

$$
\psi_{j} * f=\psi_{j} * \Lambda * \Phi * f+\sum_{k=1}^{\infty} \psi_{j} * \lambda_{k} * \psi_{k} * f .
$$

By replacing $f$ with $f\left(2^{-j}\right.$.) for $j \in \mathbb{N}$, we obtain

$$
f=\Lambda_{j} * \Phi_{j} * f+\sum_{k=j+1}^{\infty} \lambda_{k} * \psi_{k} * f .
$$

Thus

$$
\psi_{j} * f=\left(\Lambda_{j} * \Phi_{j}\right) *\left(\psi_{j} * f\right)+\sum_{k=j+1}^{\infty}\left(\psi_{j} * \lambda_{k}\right) *\left(\psi_{k} * f\right) .
$$

By Lemma 1, we know that

$$
\left|\psi_{j} * \lambda_{k}(z)\right| \leq C_{N} \frac{2^{j n} 2^{(j-k) N}}{\left(1+2^{j}|z|\right)^{a}}, \quad z \in \mathbb{R}^{n},
$$

holds for $k \geq j$ with arbitrarily large $N>0$, where $C_{N}>0$ is a constant dependent on $N$. It is also easy to see that

$$
\left|\psi_{j} * \lambda_{j}(z)\right| \leq C \frac{2^{j n}}{\left(1+2^{j}|z|\right)^{a}}, \quad z \in \mathbb{R}^{n} .
$$

By putting the last two estimates (17) and (18) into (16), we obtain for all $f \in \mathcal{S}^{\prime}\left(\mathbb{R}^{n}\right), y \in \mathbb{R}^{n}$, and $j \in \mathbb{N}$,

$$
\left|\psi_{j} * f(y)\right| \leq C_{N} \sum_{k=j}^{\infty} 2^{j n} 2^{(j-k) N} \int \frac{\left|\psi_{k} * f(z)\right|}{\left(1+2^{j}|y-z|\right)^{a}} d z .
$$


For any $r \in(0,1]$, dividing both sides of (19) by $\left(1+2^{j}|x-y|\right)^{a}$, then in the left hand side take the supremum over $y \in \mathbb{R}^{n}$, and making use of the following inequalities

$$
\begin{gathered}
\left(1+2^{j}|x-y|\right)\left(1+2^{j}|y-z|\right) \geq\left(1+2^{j}|x-y|\right), \\
\left|\psi_{k} * f(z)\right| \leq\left|\psi_{k} * f(z)\right|^{r}\left[\psi_{k, a}^{*} f(x)\right]^{1-r}\left(1+2^{k}|x-z|\right)^{a(1-r)},
\end{gathered}
$$

and

$$
\frac{\left(1+2^{k}|x-z|\right)^{a(1-r)}}{\left(1+2^{j}|x-z|\right)^{a}} \leq \frac{2^{(k-j) a}}{\left(1+2^{k}|x-z|\right)^{a r}},
$$

we finally obtain that for all $f \in \mathcal{S}^{\prime}\left(\mathbb{R}^{n}\right), x \in \mathbb{R}^{n}$ and $j \in \mathbb{N}$,

$$
\psi_{j, a}^{*} f(x) \leq C_{N} \sum_{k=j}^{\infty} 2^{(j-k) N^{\prime}} \int \frac{2^{k n}\left|\psi_{k} * f(z)\right|^{r}}{\left(1+2^{k}|x-z|\right)^{a r}} d z\left[\psi_{k, a}^{*} f(x)\right]^{1-r}
$$

where $N^{\prime}=N-a+n$ can be taken arbitrarily large.

Similarly, we can prove that for all $f \in \mathcal{S}^{\prime}(\mathbb{R})$,

$$
\begin{aligned}
\psi_{a}^{*} f(x) \leq & C_{N}\left(\int \frac{|\Psi * f(z)|^{r}}{(1+|x-z|)^{a r}} d z\left[\Psi_{a}^{*} f(x)\right]^{1-r}\right. \\
& \left.+\sum_{k=1}^{\infty} 2^{-k N^{\prime}} \int \frac{2^{k n}\left|\psi_{k} * f(z)\right|^{r}}{\left(1+2^{k}|x-z|\right)^{a r}} d z\left[\psi_{k, a}^{*} f(x)\right]^{1-r}\right) .
\end{aligned}
$$

We now fix any $x \in \mathbb{R}^{n}$ and apply Lemma 5 with

$$
\begin{gathered}
d_{j}=\psi_{j, a}^{*} f(x) \quad \text { for } j \in \mathbb{N}, \quad d_{0}=\Psi_{a}^{*} f(x), \\
b_{j}=\int \frac{2^{k n}\left|\psi_{k} * f(z)\right|^{r}}{\left(1+2^{k}|x-z|\right)^{a r}} d z \text { for } j \in \mathbb{N}, \quad \text { and } b_{0}=\int \frac{|\Psi * f(z)|^{r}}{(1+|x-z|)^{a r}} d z .
\end{gathered}
$$

We then obtain

$$
\left[\psi_{j, a}^{*} f(x)\right]^{r} \leq C_{N}^{\prime} \sum_{k=j}^{\infty} 2^{(j-k) N r} \int \frac{2^{k n}\left|\psi_{k} * f(z)\right|^{r}}{\left(1+2^{k}|x-z|\right)^{a r}} d z
$$

where $C_{N}^{\prime}=C_{N+a-n}$,

We remark that (23) also hold when $r>1$. In fact, to see this, it suffices to take (19) with $a+n$ instead of $a$, apply Hölder's inequality in $k$ and $z$, and finally deduce from (20).

Since $\frac{1}{(1+|z|)^{a r}} \in L_{1}$, by (3.9) in [9, Chapter 2], we deduce from (23) that

$$
\left[\psi_{j, a}^{*} f(x)\right]^{r} \leq C_{N}^{\prime} \sum_{k=j}^{\infty} 2^{(j-k) N r} \mathcal{M}\left(\left|\psi_{k} * f\right|^{r}\right)(x),
$$

and a similar inequality with $\psi_{j, a}^{*} f(x)$ replaced by $\Psi_{a}^{*} f(x)$.

By (24), choosing $N>\max (-s, 0)$ and applying Lemma 3 with

$$
g_{j}=2^{j s r} \mathcal{M}\left(\left|\psi_{k} * f\right|^{r}\right) \quad \text { for } j \in \mathbb{N}, \quad \text { and } g_{0}=\mathcal{M}\left(|\Psi * f|^{r}\right),
$$


we obtain that for all $f \in \mathcal{S}^{\prime}\left(\mathbb{R}^{n}\right)$

$(25)$

$$
\begin{aligned}
\left\|\Psi_{a}^{*} f\right\|_{K_{q}^{\alpha, p}}+\| & \left.\| 2^{s j} \psi_{j, a}^{*} f\right\}_{1}^{\infty} \|_{\ell_{\beta}\left(K_{q}^{\alpha, p}\right)} \\
& \lesssim\left\|\mathcal{M}_{r}(\Psi * f)\right\|_{K_{q}^{\alpha, p}}+\left\|\left\{2^{j s} \mathcal{M}_{r}\left(\psi_{j} * f\right)\right\}_{1}^{\infty}\right\|_{\ell_{\beta}\left(K_{q}^{\alpha, p}\right)},
\end{aligned}
$$

$$
\begin{aligned}
\left\|\Psi_{a}^{*} f\right\|_{\dot{K}_{q}^{\alpha, p}}+\| & \left\{2^{s j} \psi_{j, a}^{*} f\right\}_{1}^{\infty} \|_{\ell_{\beta}\left(\dot{K}_{q}^{\alpha, p}\right)} \\
& \lesssim\left\|\mathcal{M}_{r}(\Psi * f)\right\|_{\dot{K}_{q}^{\alpha, p}}+\left\|\left\{2^{j s} \mathcal{M}_{r}\left(\psi_{j} * f\right)\right\}_{1}^{\infty}\right\|_{\ell_{\beta}\left(\dot{K}_{q}^{\alpha, p}\right)}, \\
\left\|\Psi_{a}^{*} f\right\|_{K_{q}^{\alpha, p}}+\left\|\left\{2^{s j} \psi_{j, a}^{*} f\right\}_{1}^{\infty}\right\|_{K_{q}^{\alpha, p}\left(\ell_{\beta}\right)} & \\
& \lesssim\left\|\mathcal{M}_{r}(\Psi * f)\right\|_{K_{q}^{\alpha, p}}+\left\|\left\{2^{j s} \mathcal{M}_{r}\left(\psi_{j} * f\right)\right\}_{1}^{\infty}\right\|_{K_{q}^{\alpha, p}\left(\ell_{\beta}\right)},
\end{aligned}
$$

and

$\left(26^{\prime}\right)$

$$
\begin{aligned}
\left\|\Psi_{a}^{*} f\right\|_{\dot{K}_{q}^{\alpha, p}}+\| & \left\{2^{s j} \psi_{j, a}^{*} f\right\}_{1}^{\infty} \|_{\dot{K}_{q}^{\alpha, p}\left(\ell_{\beta}\right)} \\
& \lesssim\left\|\mathcal{M}_{r}(\Psi * f)\right\|_{\dot{K}_{q}^{\alpha, p}}+\left\|\left\{2^{j s} \mathcal{M}_{r}\left(\psi_{j} * f\right)\right\}_{1}^{\infty}\right\|_{\dot{K}_{q}^{\alpha, p}\left(\ell_{\beta}\right)},
\end{aligned}
$$

where we used the notation $\mathcal{M}_{r}(g)=\left(\mathcal{M}\left(|g|^{r}\right)\right)^{1 / r}$.

For (25) and $\left(25^{\prime}\right)$, we choose $r$ so that $n / a<r<\beta$ and $-n r / q<r \alpha<$ $n(1-r / q)$. By Lemma 4 , we have $(14)$ and $\left(14^{\prime}\right)$.

For (26) and $\left(26^{\prime}\right)$, we choose $r$ so that $n / a<r<\min (q, \beta)$ and $-n r / q<r \alpha<n(1-r / q)$. By Lemma 4 , we have (15) and $\left(15^{\prime}\right)$.

Step 3 . We will prove that $(4),\left(4^{\prime}\right),(5),\left(5^{\prime}\right)$ can follow from $(10),\left(10^{\prime}\right)$, (11), $\left(11^{\prime}\right)$ and $(14),\left(14^{\prime}\right),(15),\left(15^{\prime}\right)$. For instance, we check (4).

The left inequality in (4) is proved by the chain of estimates

$$
\text { the left side of }(4) \lesssim\left\|A_{a}^{*} f\right\|_{K_{q}^{\alpha, p}}+\left\|\left\{2^{j s} \theta_{j} * f\right\}\right\|_{\ell_{\beta}\left(K_{q}^{\alpha, p}\right)} \lesssim\|f\|_{K_{q}^{\alpha, p} B_{\beta}^{s}} \text {, }
$$

here we first used (10) with $\Phi=A, \varphi=\theta$, and then applied (15) with $\Psi=A, \psi=\theta$.

The right inequality in (4) is proved by another chain

$$
\begin{aligned}
\|f\|_{K_{q}^{\alpha, p} B_{\beta}^{s}} & \lesssim\left\|A_{a}^{*} f\right\|_{K_{q}^{\alpha, p}}+\left\|\left\{2^{j s} \theta_{j} * f\right\}\right\|_{\ell\left(K_{q}^{\alpha, p}\right)} \\
& \lesssim\left\|\Psi_{a}^{*} f\right\|_{K_{q}^{\alpha, p}}+\left\|\left\{2^{j s} \psi_{j, a}^{*} f\right\}\right\|_{\ell_{\beta}\left(K_{q}^{\alpha, p}\right)} \\
& \lesssim R H S(4),
\end{aligned}
$$

where the the first inequality is obvious, the second is (10) with $\Phi=\Psi, \varphi=$ $\psi$, and $A$ and $\theta$ instead of $\Psi$ and $\psi$ in the left hand side. Finally, the third inequality is (15).

This completes the proof.

Acknowledgements. The author would like to thank the referee for his suggestions. 
30 Equivalent norms of Herz-type Besov and Triebel-Lizorkin spaces

\section{References}

[1] J. Alvarez, M. Guzmn-Partida and J. Lakey, Spaces of bounded $\Lambda$-central mean oscillation, Morrey spaces and $\Lambda$-central Carleson measures, Collect. Math., 51 (2000), 1-47.

[2] A. Baernstein and E. Sawyer, Embedding and multiplier theorems for $H^{p}\left(\mathbb{R}^{n}\right)$, Memoirs Amer. Math. Soc., 59 (318); Amer. Math. Soc., Providence R.I., 1985.

[3] J. Betancor, Herz-type Hardy space and Bochner-Riesz means on the Hankel setting, Preprint, 2000.

[4] A. Beurling, Construction and analysis of some convolution algebras, Ann. Inst. Fourier Grenoble, 14 (1964), 1-32.

[5] Y. Chen and K. Lau, On some new classes of Hardy spaces, J. Funct. Anal., 84 (1989), 255-278.

[6] S. Lu and D. Yang, The weighted Herz-type Hardy space and its applications, Sci. in China (Ser. A), 38 (1995), 662-673.

[7] S. Lu and D. Yang, Herz-type Sobolev and Bessel potential space and their applications, Sci. in China (Ser. A), 40 (2) (1997), 113-129.

[8] V. Rychkov, On a theorem of Bui, Paluszyński and Taibleson, Proc. Steklov Inst. Math., 227 (1999), 280-292.

[9] E. Stein and G. Weiss, Introduction to Fourier analysis on Euclidean spaces, Princeton Univ. Press, Princeton, NJ, 1971.

[10] L. Tang and D. Yang, Boundedness of vector-valued operators on weighted Herz space, Approx. Theory Appl., 16 (2) (2000), 58-70.

[11] H. Triebel, Theory of function spaces, Basel, Birkhäuser, 1983.

[12] H. Triebel, Theory of function spaces II, Basel, Birkhäuser, 1992.

[13] H. Triebel, Fractals and spectra: related to Fourier analysis and function spaces, Basel, Birkhäuser, 1997.

[14] H. Triebel, The structure of functions, Basel, Birkhäuser, 2001.

[15] J. Xu and D. Yang, Herz-type Triebel-Lizorkin spaces, Acta Math. Sin. (Engl. Ser.), to appear.

[16] J. Xu and D. Yang, Applications of Herz-type Triebel-Lizorkin spaces, Acta Math. Scientia, 23 (B) (2003), 328-338.

[17] J. Xu, A discrete characterization of the Herz-type Triebel-Lizorkin spaces and its application, Acta Math. Scientia (Engl. Ser.), to appear.

[18] J. Xu, Some properties on Herz-type Besov spaces (in Chinese), J. Hunan Univ. (Natural Sci.), 30 (5) (2003), 75-78. 
[19] J. Xu, Pointwise multipliers of Herz-type Besov spaces and their applications, Appl. Math., 17 (1) (2004), 115-121.

Department of Mathematics

Hunan Normal University

Changsha 410081

People's Republic of China

and

Department of Mathematics

Changsha University of Science and Technology

Changsha 410076

People's Republic of China

(E-mail : jshixu@yahoo.com.cn) 


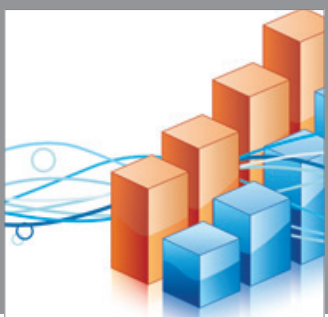

Advances in

Operations Research

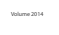

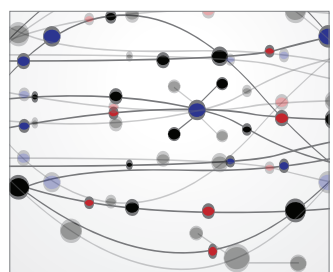

\section{The Scientific} World Journal
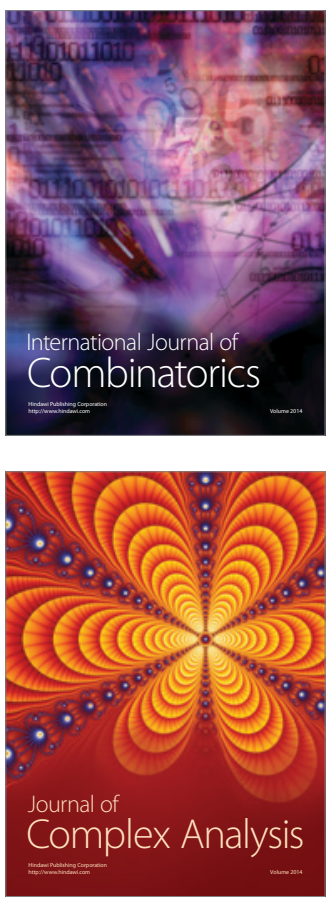

International Journal of

Mathematics and

Mathematical

Sciences
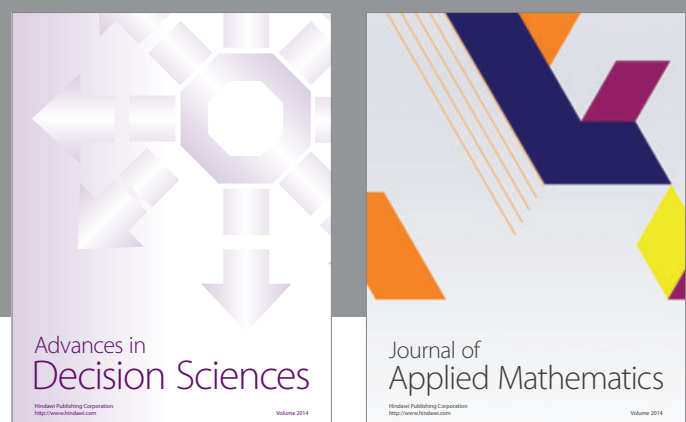

Journal of

Applied Mathematics
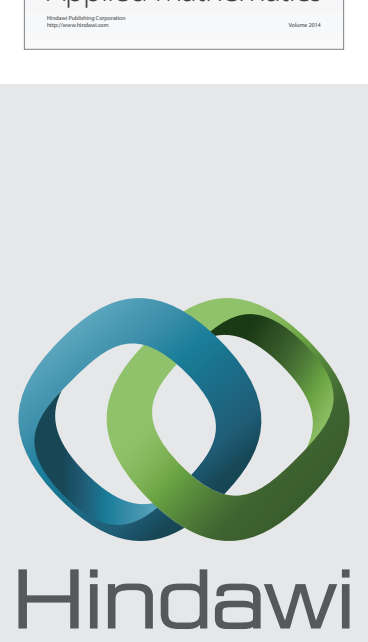

Submit your manuscripts at http://www.hindawi.com
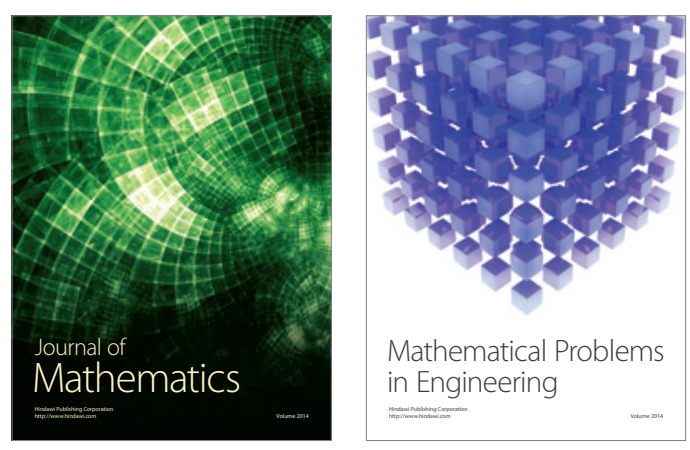

Mathematical Problems in Engineering
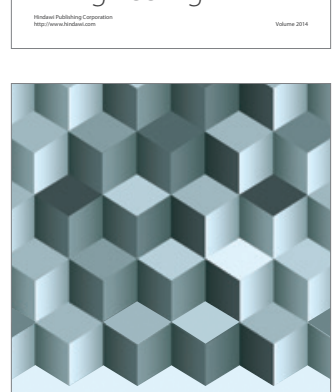

Journal of

Function Spaces
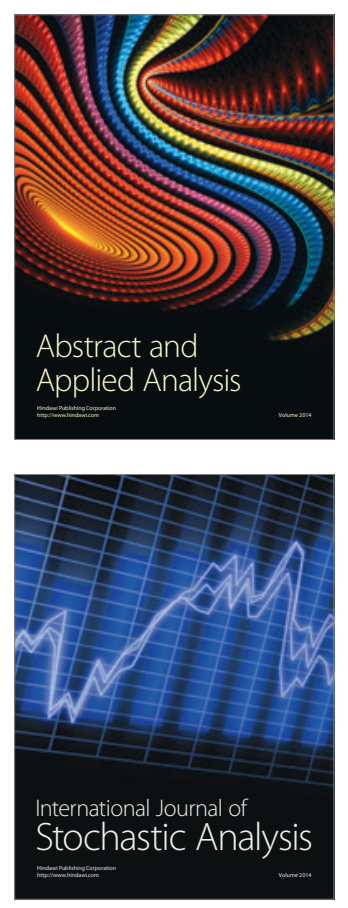

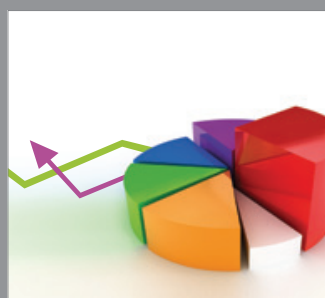

ournal of

Probability and Statistics

Promensencen
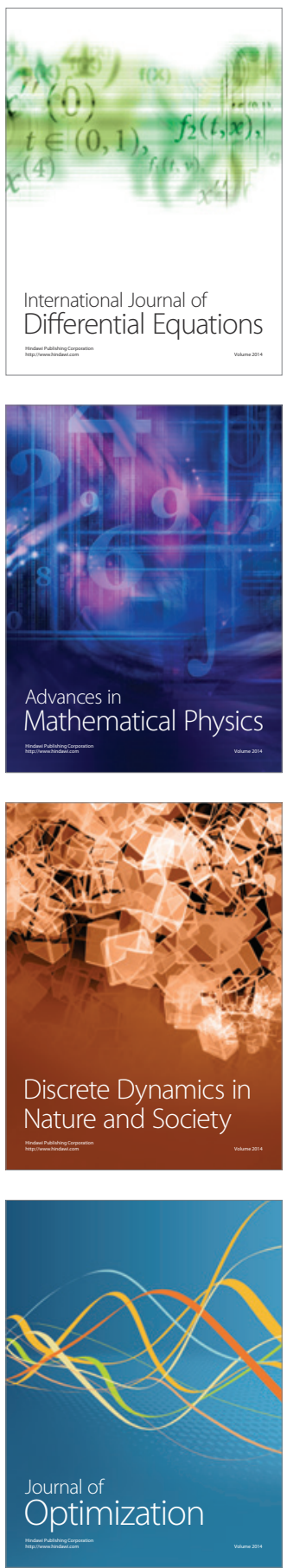